\title{
Experimental Monte Carlo Mistuning Assessment of Bladed Disk Vibration Using Forcing Variations
}

\author{
Jia Li* and Matthew P. Castanier ${ }^{\dagger}$ \\ University of Michigan, Ann Arbor, Michigan 48109-2125 \\ Christophe Pierre f $^{\ddagger}$ \\ McGill University, Montreal, Quebec H3A 2K6, Canada \\ Steven L. Ceccio ${ }^{\S}$ \\ University of Michigan, Ann Arbor, Michigan 48109-2125
}

\begin{abstract}
It is known that the vibration response of bladed disks in turbine engine rotors can be extremely sensitive to small, random blade-to-blade variations, or mistuning. However, it is cumbersome to assess the effects of random mistuning or validate numerical predictions for a population of mistuned bladed disks using experimental methods. In this work, as an alternative approach for performing vibration testing of many mistuning patterns, it is proposed that varying the external forcing function provided to the blades can be used to mimic the influence of structural blade property mistuning on the vibration response. Because it is much easier and more efficient to vary the external excitation than to physically alter the blades, this opens the possibility of running an experimental analog of a Monte Carlo simulation. This approach, referred to as an experimental Monte Carlo mistuning assessment, is explored and validated by comparing simulations and test data for a 24bladed disk. The experimental Monte Carlo results are found to have excellent agreement with numerical Monte Carlo simulation results for a case of small standard deviation of mistuning.
\end{abstract}

\section{Introduction}

$\mathrm{T}^{\mathrm{T}}$ is well known that the vibration response of bladed disks found in turbine engine rotors can be extremely

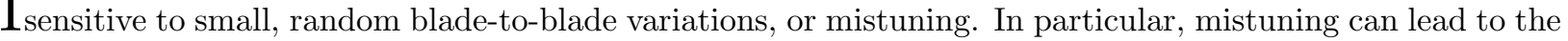
vibration energy becoming localized in a few blades, causing significant increases in maximum blade vibration amplitudes and stress levels. ${ }^{1-6}$ In order to predict the mistuned forced response in a computationally efficient manner, several reduced-order modeling techniques have been developed. ${ }^{7-10}$ These methods can be used to generate reduced-order models that are sufficiently compact that Monte Carlo simulations can be performed to estimate the statistics of the forced response for a population of mistuned rotors. In these Monte Carlo simulations, ${ }^{11-13}$ mistuning patterns are assigned with a pseudo-random number generator, and a certain physical or modal property of each blade (e.g., Young's modulus or blade-alone natural frequency) is altered in the model according to its assigned mistuning value.

However, it is much more difficult to assess the effects of random mistuning or validate numerical predictions using experimental methods. In particular, imposing specific mistuning patterns on a physical system is challenging. Although adding mistuning masses is an effective means of controlling mistuning in bench tests, it can be time-consuming and cumbersome to precisely manufacture and attach the individual mistuning masses. As a result, relatively few mistuning patterns can be tested using this approach. ${ }^{14,15}$

* Graduate Student Research Assistant, Department of Mechanical Engineering. Student Member of AIAA.

${ }^{\dagger}$ Associate Research Scientist, Department of Mechanical Engineering. Senior Member of AIAA.

${ }^{\ddagger}$ Dean, Faculty of Engineering. Senior Member of AIAA.

$\S$ Professor, Department of Mechanical Engineering. 
In this work, as an alternative approach for vibration testing of many mistuning patterns, it is proposed that varying the external forcing function provided to the blades can be used to mimic the influence of structural blade property mistuning on the vibration response. Because it is much easier and more efficient to vary the external excitation than to physically alter the blades, this opens the possibility of running an experimental analog of a Monte Carlo simulation. In the following sections, the feasibility of this approach, referred to as an experimental Monte Carlo mistuning assessment, is explored. First, a recently developed reduced-order modeling technique, called the component mode mistuning (CMM) method, is used to generate the equations of motion for the system. Then, the modified forcing function required to mimic a given blade mistuning pattern is derived. Finally, the probability density function (PDF) and cumulative distribution function $(\mathrm{CDF})$ predicted by numerical Monte Carlo simulations are compared to those estimated from the new experimental approach.

\section{Theory}

\section{A. Mistuning identification and model updating based on the CMM method}

In the CMM method, ${ }^{10}$ a selected set of system modes of the tuned bladed disk is used as a modeling basis. ${ }^{8}$ The diagonal matrix of eigenvalues for this set of tuned system modes is $\boldsymbol{\Lambda}^{S}$. Individual blade mistuning is modeled as the deviation of mistuned cantilevered-blade modal stiffness values from the tuned values. The diagonal matrix containing the mistuning values for all of the blades in cantilevered-blade modal coordinates is $\delta \boldsymbol{\Lambda}^{C B}$. This individual blade mistuning is then projected to the reduced-order model of the system by relating the cantilevered-blade (component) mode shapes to the blade part of the system mode shapes. In particular, a matrix of modal participation factors, $\mathbf{Q}^{C B}$, is generated, which defines the transformation of blade mistuning from cantilevered-blade modal coordinates to the generalized coordinates of the reducedorder model.

In addition, the CMM method has recently been extended by the authors to perform both mistuning identification and reduced-order model updating. ${ }^{16-18}$ A sensitivity study was performed to consider the influence of errors in modeling parameters and measured data on the mistuning identification results. It was observed that the identification results are most sensitive to errors in the tuned system eigenvalues. In order to compensate for these errors, it was proposed that the "cyclic modeling error" could be identified from the test data assuming the mistuning in the actual system has a mean value of zero. ${ }^{16,17}$ The cyclic modeling error, $\delta \boldsymbol{\Lambda}^{C}$, is defined as the difference between the tuned system eigenvalues predicted from the finite element model and those identified for the actual bladed disk. Thus, after identifying the blade mistuning and the cyclic modeling error, the modal stiffness matrix of the updated model is:

$$
\mathbf{K}^{U}=\boldsymbol{\Lambda}^{S}+\delta \boldsymbol{\Lambda}^{C}+\mathbf{Q}^{C B^{T}} \delta \boldsymbol{\Lambda}^{C B} \mathbf{Q}^{C B}
$$

Then, the equations of motion can be expressed in tuned system modal coordinates as:

$$
\left[-\omega^{2} \mathbf{I}+(1+j \gamma) \mathbf{K}^{U}\right] \mathbf{q}=\mathbf{f}
$$

where $\omega$ is the excitation frequency, $j=\sqrt{-1}, \gamma$ is the structural damping factor, $\mathbf{q}$ is the response vector, and $\mathbf{f}$ is the forcing vector. Note that the size of the reduced-order model is equal to the number of selected tuned system modes.

\section{B. Formulation for modified forces to mimic the effects of structural blade mistuning}

In order to perform an experimental Monte Carlo mistuning assessment, the required forcing variations are determined as follows. Suppose that a given mistuning pattern, $\delta \boldsymbol{\Lambda}_{i n t}^{C B}$, is intentionally imposed on a test specimen and a certain engine order excitation vector, $\mathbf{f}_{E}$, is applied as the forcing function. The equations of motion are then:

$$
\left[-\omega^{2} \mathbf{I}+(1+j \gamma)\left(\mathbf{K}^{U}+\mathbf{Q}^{C B^{T}} \delta \boldsymbol{\Lambda}_{\text {int }}^{C B} \mathbf{Q}^{C B}\right)\right] \mathbf{q}_{m}=\mathbf{f}_{E}
$$

where $\mathbf{q}_{m}$ is the mistuned vibration response for the given engine order excitation. Now, assume that the same response could be achieved by mistuning the forcing vector instead of the structural system. The corresponding equations of motion are: 


$$
\left[-\omega^{2} \mathbf{I}+(1+j \gamma) \mathbf{K}^{U}\right] \mathbf{q}_{m}=\mathbf{f}_{m}
$$

where $\mathbf{f}_{m}$ is the modified (or mistuned) forcing vector. From Eq. (3), the mistuned response can be expressed in terms of the given mistuning pattern and engine order excitation:

$$
\mathbf{q}_{m}=\left[-\omega^{2} \mathbf{I}+(1+j \gamma)\left(\mathbf{K}^{U}+\mathbf{Q}^{C B^{T}} \delta \mathbf{\Lambda}_{i n t}^{C B} \mathbf{Q}^{C B}\right)\right]^{-1} \mathbf{f}_{E}
$$

Substituting Eq. (5) into Eq. (4), the mistuned forcing vector is solved as:

$$
\mathbf{f}_{m}=\left[-\omega^{2} \mathbf{I}+(1+j \gamma) \mathbf{K}^{U}\right]\left[-\omega^{2} \mathbf{I}+(1+j \gamma)\left(\mathbf{K}^{U}+\mathbf{Q}^{C B^{T}} \delta \boldsymbol{\Lambda}_{\text {int }}^{C B} \mathbf{Q}^{C B}\right)\right]^{-1} \mathbf{f}_{E}
$$

Note that the mistuned forcing is frequency-dependent.

\section{Estimation of the three-parameter Weibull distribution}

For mistuned bladed disk vibration, the variable of interest is the maximum blade response for any blade in the system. Therefore, the theory of the statistics of extremes ${ }^{19,20}$ can be used to accelerate the Monte Carlo simulations. In particular, the distribution of the maximum blade response will tend toward a Weibull distribution. The PDF of a three-parameter Weibull distribution for maximum values is:

$$
\mathrm{PDF}=\frac{\beta}{\delta}\left(\frac{\lambda-x}{\delta}\right)^{\beta-1} e^{-\left(\frac{\lambda-x}{\delta}\right)^{\beta}}
$$

where $\delta, \beta$ and $\lambda$ are the scale, shape, and location parameters, respectively, and $x$ is the variable of interest (i.e., the maximum blade response amplitude or magnification factor). The location parameter represents the upper limit of the value $x$. In this paper, it is taken to be the approximate maximum response magnification factor calculated by Whitehead, ${ }^{6}$ which is

$$
\lambda=\frac{1}{2}(1+\sqrt{N})
$$

where $N$ is the number of blades in the bladed disk. In a numerical simulation, by using this approximate value for the upper limit, the other two parameters of the Weibull distribution can be estimated using data obtained from relatively few Monte Carlo realizations. ${ }^{11,13}$ In the case of an experimental Monte Carlo assessment, this corresponds to running relatively few tests with mistuned excitation fields as specified in Eq. (6).

\section{Experimental Specimen and Excitation System}

A 24-bladed disk was examined for this study, and it is shown in Figs. 1 and 2. Figure 1 shows the finite element model and Fig. 2 shows the actual bladed disk, which was manufactured to extremely tight tolerances. ${ }^{14}$ This bladed disk, which is referred to as the validation rotor, has been used to experimentally validate the CMM approach and the reduced-order model updating method discussed above. ${ }^{18}$ In this study, the frequency range $2110-2170 \mathrm{~Hz}$ was considered, which excites the first flexural bending family of blade-dominated system modes.

Acoustic excitation was provided to the blades by an array of speakers, ${ }^{12,21,22}$ as seen in Fig. 2. Behind each blade, there is a round speaker mounted in a parallel position with the surface of the blade in order to apply an acoustic force. These speakers are driven by a series of Hewlett-Packard 8904 Multifunction Synthesizers, each with two independent but phase-synchronized channels. The speakers were carefully calibrated using white noise excitation first. Then, the speakers were mounted on the plastic fixtures, and a sinusoidal signal was sent to each speaker, one at a time. A calibrated microphone was used to record the sound pressure level at a fixed distance from the speaker, similar to the distance to the individual blade when it is mounted on the fixture. The amplitude and phase of the excitation signal were adjusted in order to achieve a flat frequency response of the sound pressure level produced by the speaker. This process was digitally controlled and it was repeated, in turn, for each speaker on the fixture. 


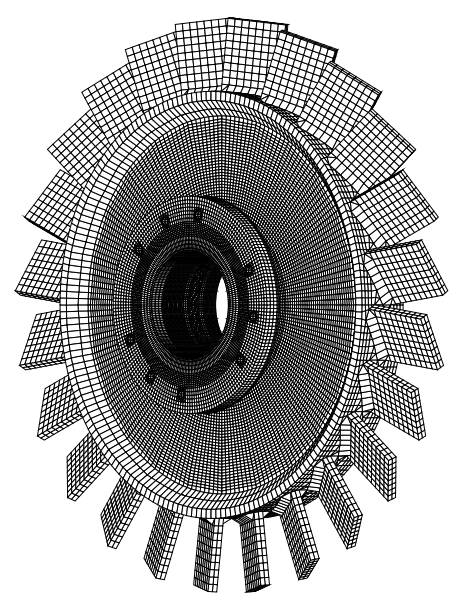

Figure 1. Finite element model of the bladed disk

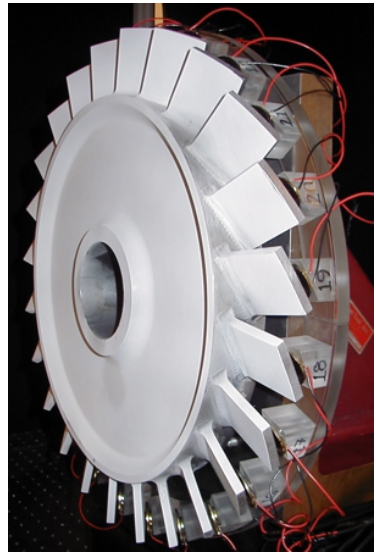

Figure 2. Actual bladed disk used in the experiment, shown with the acoustic excitation system

To achieve engine order excitation, all the speakers are driven simultaneously to deliver forces of the same amplitude, but with a certain phase lag between adjacent speakers:

$$
\phi=\frac{2 \pi C}{N}
$$

where $C$ is the engine order of the excitation. Thus, the force for blade number $n$ can be written as:

$$
f_{n}=f_{0} \cos (\omega t+(n-1) \phi)
$$

where $f_{0}$ is the force amplitude. Thus any engine order excitation can be obtained by simply changing the phase lag. The traveling-wave excitation system not only simulates the forcing experienced by a rotating bladed disk in an engine, but it also provides non-contacting excitation. ${ }^{14,22}$

For the experimental system used in this investigation, the amplitude of the excitation was controlled independently for each speaker. However, only 12 channels were available for independent phase control. For the validation rotor with 24 blades, this was sufficient to deliver engine order excitation. This is because, for any engine order, the phase of speaker $n+12$ is either 0 or 180 degrees behind the phase of speaker $n$. Therefore, specific phase values were delivered to blades $1-12$, and the same or inverted signals were delivered to blades 13-24, respectively. However, when the "mistuned" excitation of Eq. (6) is used, this phase relationship no longer holds, and individual phase control would be needed to deliver the mistuned excitation field precisely. The lack of independent phase control for all speakers thus introduced some experimental error.

\section{Computational Monte Carlo Simulation}

In order to perform computational Monte Carlo simulations, mistuning identification and model updating techniques were first applied to the validation rotor experimentally to obtain the updated CMM model for the first flexural bending family of blade-dominated system modes. The standard deviation of identified blade stiffness mistuning was $0.29 \%$. Then, for a given value of standard deviation of mistuning, 1000 random mistuning patterns generated from a uniform distribution were added to the updated CMM model. For each simulated mistuned system, a frequency sweep was performed, and the highest peak response value of any blade in the mistuned system was determined. This value was divided by the peak response value of the tuned system to yield a magnification factor. Then the Weibull distribution of the magnification factor was estimated from the data. This process was performed for engine orders 6-12.

The Monte Carlo simulation results for engine orders 8 and 12 are depicted in Figs. 3 and 4 . It is seen that the magnification factor can be very sensitive to the mistuning level for small values of standard deviation. However, the case of engine order 8 excitation shows smoother slope than engine order 12 for small mistuning levels. This was also found to be true while comparing to engine orders 6,7 , and 9-11, although the results for those engine orders are not shown here. Therefore, engine order 8 excitation was chosen for conducting the experimental Monte Carlo assessments. 


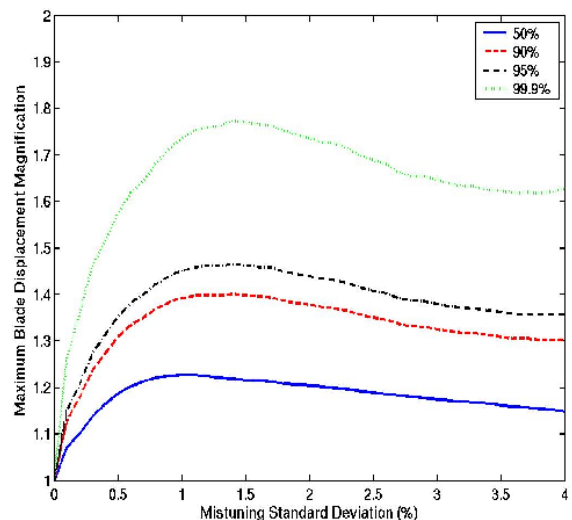

Figure 3. Monte Carlo simulation results for blade amplitude magnification, engine order 8

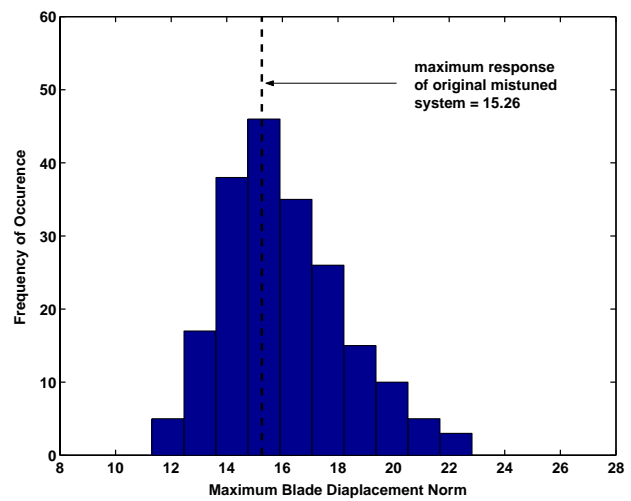

Figure 5. Histogram of the maximum blade response amplitude obtained by Monte Carlo simulation, based on $\mathbf{2 0 0}$ mistuning patterns generated from a uniform distribution with standard deviation $=0.1 \%$

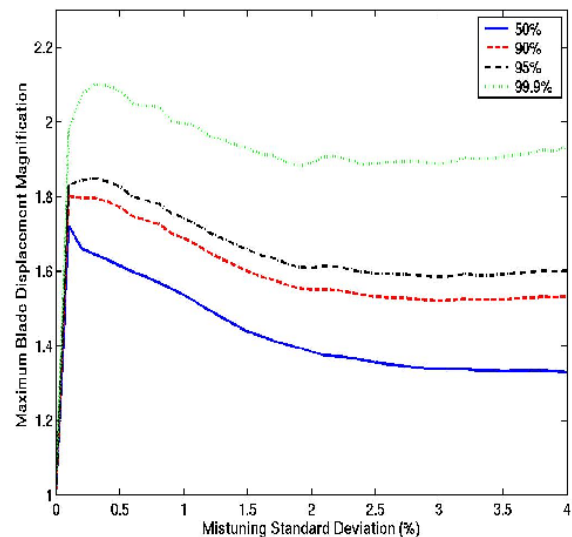

Figure 4. Monte Carlo simulation results for blade amplitude magnification, engine order 12

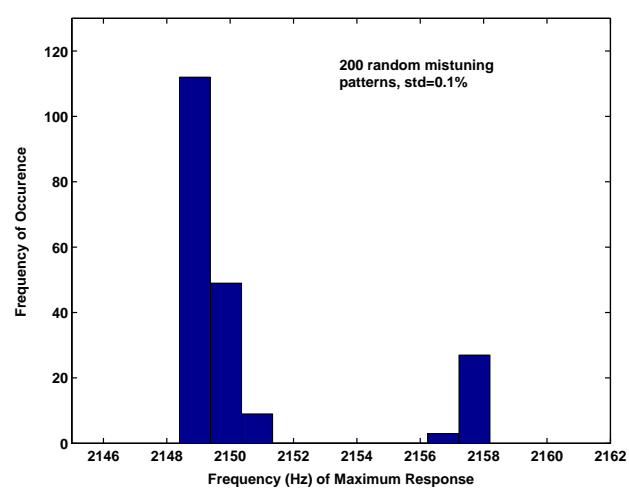

Figure 6. Histogram of the frequencies at which the maximum response amplitudes occurred, based on 200 mistuning patterns generated from a uniform distribution with standard deviation $=0.1 \%$

As mentioned above, the experimental setup had entirely independent control over the amplitudes of external forces but not the phases. Therefore, it was desired to keep the mistuned part of the force used in the experiment sufficiently small so that no dramatic effect on the phase relative to engine order 8 excitation was required. For this reason, a mistuning standard deviation of $0.1 \%$ was chosen.

Focusing on a mistuning level of $0.1 \%, 200$ random mistuning patterns were added to the updated CMM model, and the maximum blade response amplitudes were calculated for engine order 8 in the range 2145$2160 \mathrm{~Hz}$. The histogram of maximum mistuned blade response amplitudes is shown in Fig. 5, and the resonant frequency distribution is depicted in Fig. 6. Based on these results, the investigated frequency range was selected as $2147.2-2152.5 \mathrm{~Hz}$ for the experimental work, in order to reduce the required testing time.

\section{Experimental Monte Carlo Mistuning Assessment}

In all of the following experiments, the tuned force needed on the right-hand side of Eq. (6) was chosen as engine order 8 excitation, and the investigated frequency range was $2147.2-2152.5 \mathrm{~Hz}$ with a step size of 0.1 Hz. The raw measured results obtained with a Scanning Laser Doppler Vibrometer were the displacements at the tips of the blades excited by mistuned forces, and the maximum blade response amplitude was found for each mistuning pattern. These values were later divided by the maximum blade response of the rotor 
subject to pure engine order 8 excitation (the "tuned" case) to obtain the magnification factors as the input data for the Weibull distribution. Using the three-parameter Weibull distribution given in Eq. (7), the PDF and CDF were calculated from the experimental data. The test results were then compared to numerical predictions by applying the same group of mistuning patterns to the updated CMM model and performing the same Weibull distribution fit to calculate the PDF and CDF.

\section{A. Experimental Trial 1}

The first experimental trial was run for a set of 80 mistuning patterns. Figure 7 shows the PDF and CDF results for the first 20 mistuning patterns tested, which match fairly well with the predictions. For example, the CDF from the Monte Carlo simulation indicates that $99.9 \%$ of the magnification factors are expected to be lower than 1.6, which is corroborated by the experimental results. The capability of forecasting such a high-percentile response is especially useful in assessing the durability and reliability of bladed disks. Figure 8 shows the PDF and CDF results for the first 40 mistuning patterns tested. There is excellent agreement between the numerical and experimental results.

However, as the number of mistuning patterns was increased to 60 and then to 80, the experimental Monte Carlo results started to deviate from the numerical simulation results, as seen in Figs. 9 and 10. It is believed that this was due to a breakdown of the temperature control in the experimental laboratory, which led to inconsistent room temperatures for the last 40 patterns of this first trial. The room temperature should be kept consistent, since the blade displacements being measured are on the micron level. Nevertheless, note that there is still good agreement for the high-percentile values (e.g., 99.9th percentile) of the magnification factors. This indicates that the experimental Monte Carlo assessment shows great promise in terms of providing a robust prediction of the worst-case mistuning effects.

\section{B. Experimental Trial 2}

For a second experimental trial, another 80 random mistuning patterns were run for the validation rotor. This time, the experimental environment was kept consistent throughout the testing. The PDF and CDF results from trial 2 are depicted in Figs. 11-14. It can be seen that both the PDF and CDF results match well with the predictions, especially when all 80 mistuning patterns are considered. Regarding the discrepancies seen in Figs. 12 and 13, the main source of error is most likely the lack of precise phase control for all speakers. However, unlike the results of trial 1, these results seem to indicate that the experimental and computational results are starting to converge after 80 mistuning patterns, and it is expected that the agreement would improve with more patterns.

\section{Conclusions}

An innovative method for experimental Monte Carlo assessment of mistuned bladed disks has been established. The blade mistuning was recast in the equations of motion as modified external forces. This implies that various mistuning patterns can be imposed in a test environment by varying the external excitation rather than making structural changes. This approach was then validated with a series of tests performed on a 24-blade validation rotor. For a case with $0.1 \%$ standard deviation of the implemented mistuning, the experimental results matched the numerical predictions quite well. The match was especially good for high-percentile response values, which indicates that this approach might be useful for assessing the safety and reliability of a bladed disk.

The main limitation for the application of the technique in this initial study was the lack of individual phase control over each speaker that provides external excitation to each blade. As a result, only mistuning patterns generated from a distribution with a small standard deviation could be investigated in this work. However, if one had independent phase and amplitude control for each speaker in the acoustic excitation system, this restriction to small mistuning levels would be alleviated. Moreover, compared to previous experimental mistuning methods such as attaching masses to the blades, this new approach is more flexible and efficient. 

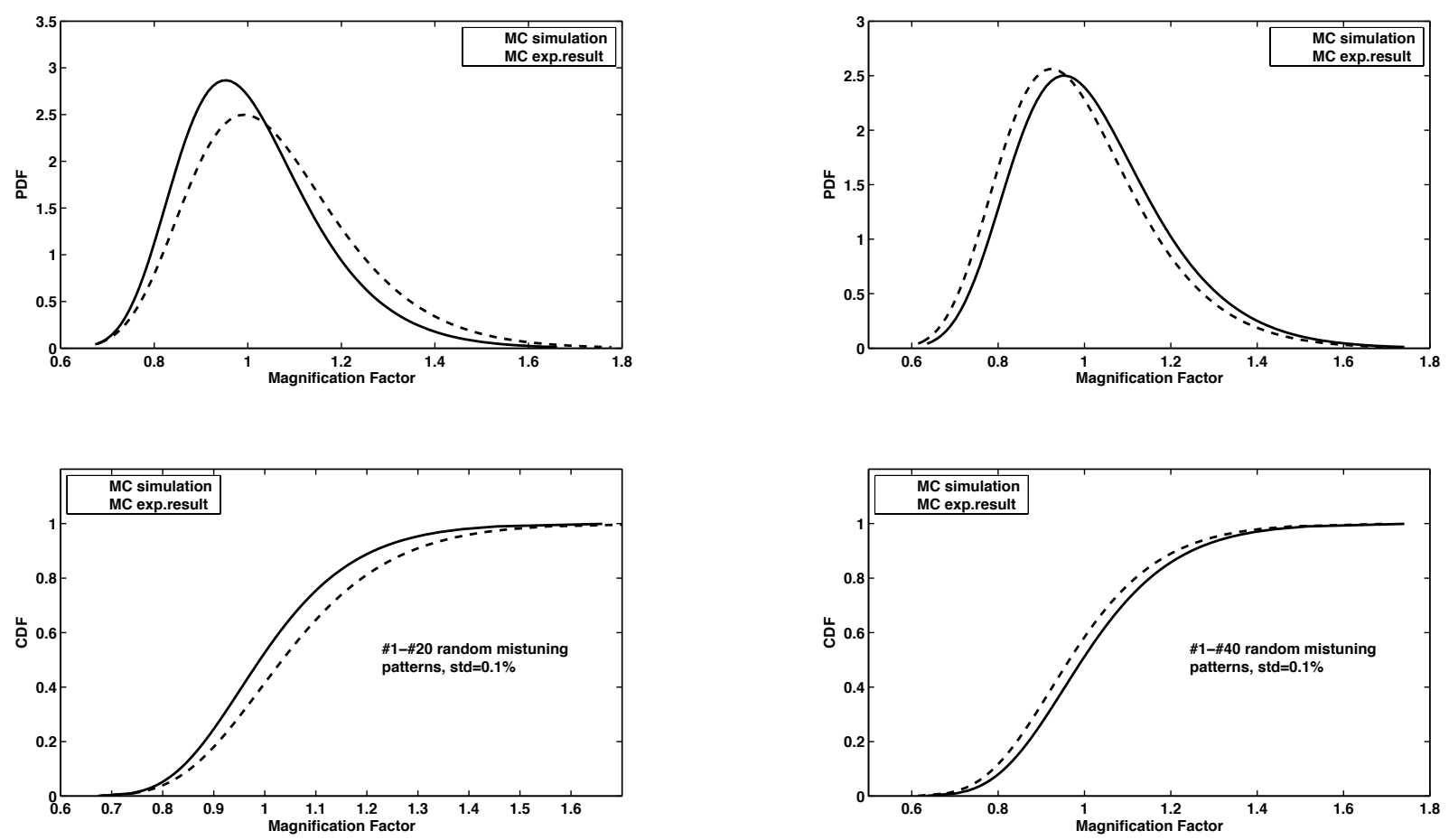

Figure 7. Trial 1, 20 mistuning patterns

Figure 8. Trial 1, 40 mistuning patterns
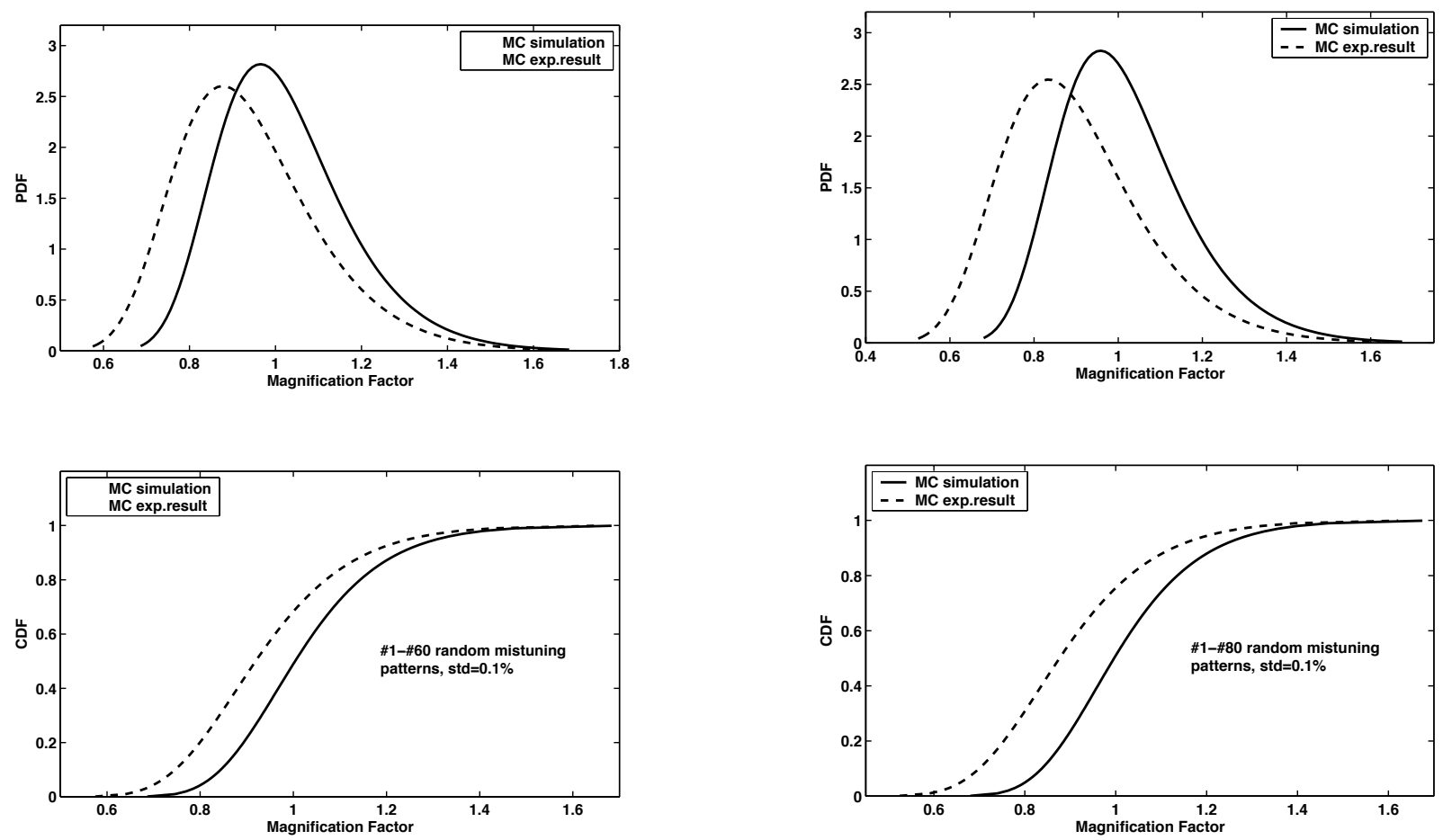

Figure 9. Trial 1, 60 mistuning patterns

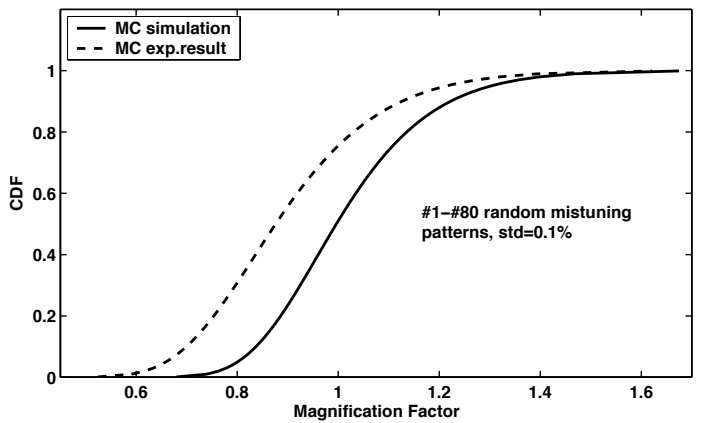

Figure 10. Trial 1, 80 mistuning patterns 

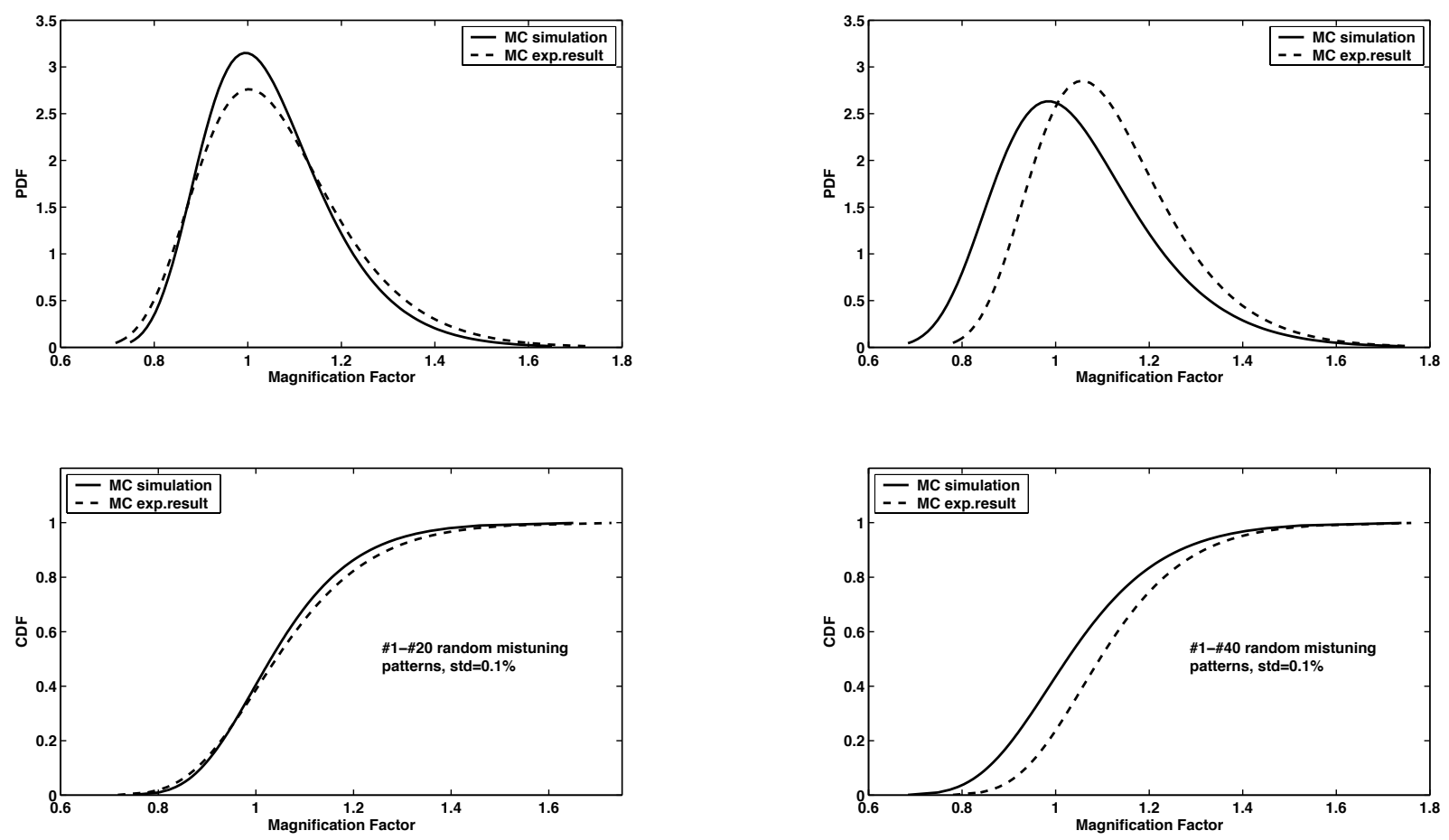

Figure 11. Trial 2, 20 mistuning patterns

Figure 12. Trial 2, 40 mistuning patterns
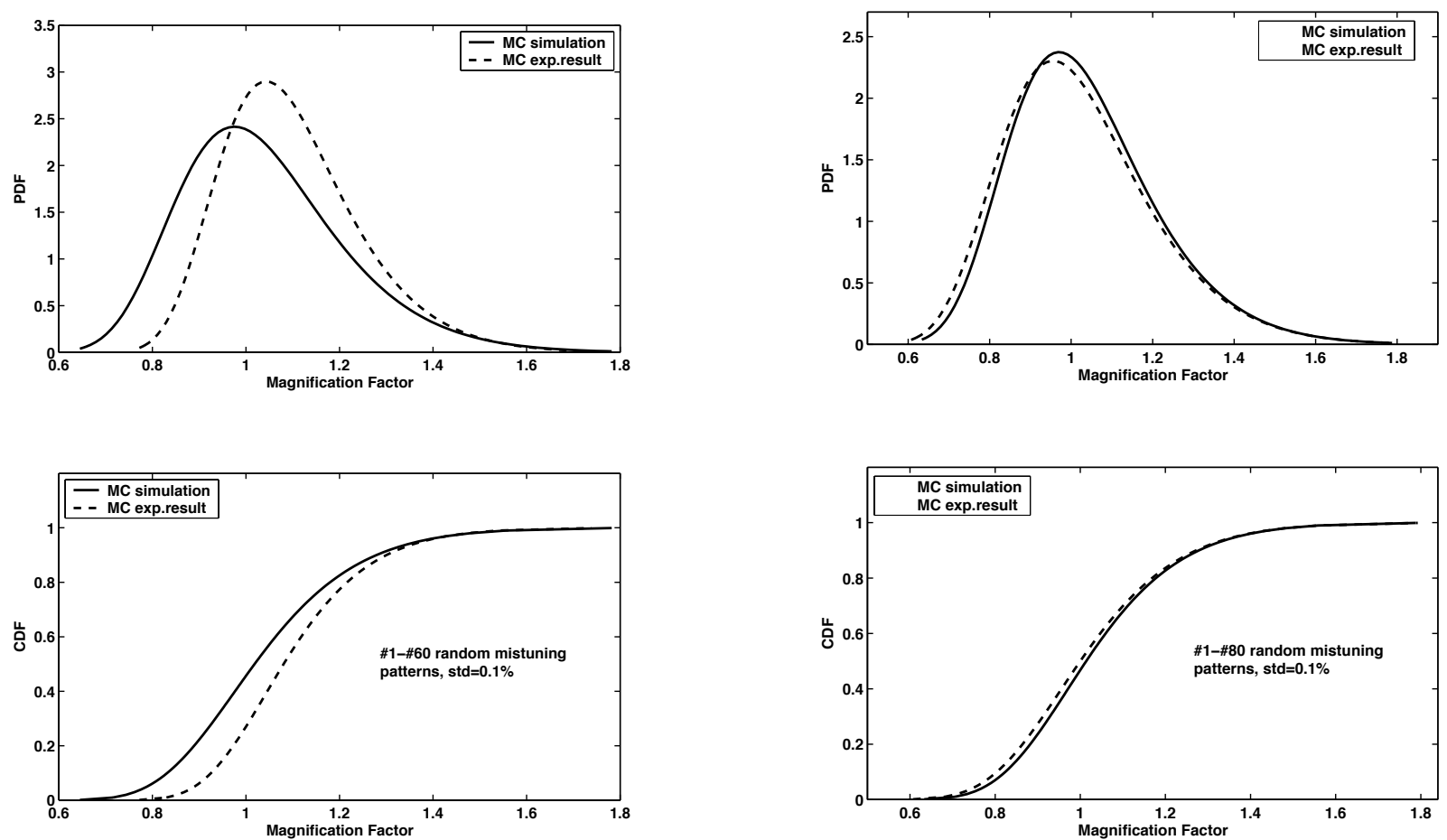

Figure 13. Trial 2, 60 mistuning patterns

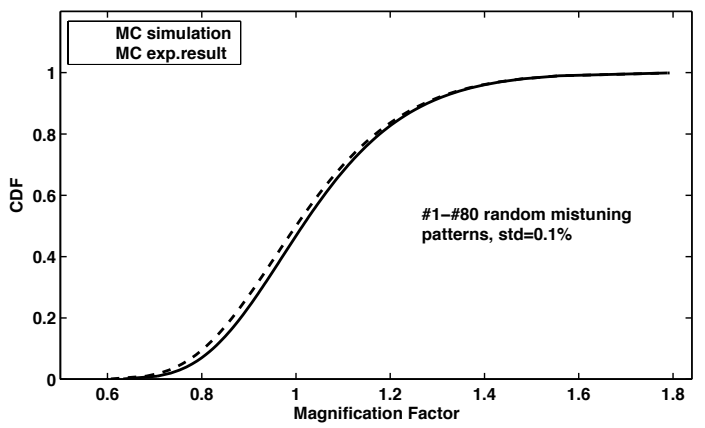

Figure 14. Trial 2, 80 mistuning patterns 


\section{Acknowledgments}

This work was sponsored by the Air Force Office of Scientific Research Structural Mechanics Program, with Capt. Clark Allred as the program manager. The support of AFOSR is gratefully acknowledged. The authors would also like to thank Dr. Sang-Ho Lim for introducing the concept of experimental Monte Carlo mistuning assessment.

\section{References}

${ }^{1}$ Whitehead, D. S., "Effect of Mistuning on the Vibration of Turbomachine Blades Induced by Wakes," Journal of Mechanical Engineering Science, Vol. 8, No. 1, 1966, pp. 15-21.

${ }^{2}$ Dye, R. C. F. and Henry, T. A., "Vibration Amplitudes of Compressor Blades Resulting From Scatter in Blade Natural Frequencies," Journal of Engineering for Power, Vol. 91, No. 3, 1969, pp. 182-188.

${ }^{3}$ Ewins, D. J., "The Effects of Detuning Upon the Forced Vibrations of Bladed Disks," Journal of Sound and Vibration, Vol. 9, No. 1, 1969, pp. 65-79.

${ }^{4}$ Griffin, J. H. and Hoosac, T. M., "Model Development and Statistical Investigation of Turbine Blade Mistuning," Journal of Vibration, Acoustics, Stress and Reliability in Design, Vol. 106, 1984, pp. 204-210.

${ }^{5}$ Ottarsson, G. and Pierre, C., "On the Effects of Interblade Coupling on the Statistics of Maximum Forced Response Amplitudes in Mistuned Bladed Disks," Proceedings of the 36th AIAA/ASME/ASCE/AHS/ASC Structures, Structural Dynamics and Materials Conference, Vol. 5, AIAA, New York, April 1995, pp. 3070-3078.

${ }^{6}$ Whitehead, D. S., "The Maximum Factor by Which Forced Vibration of Blades Can Increase Due to Mistuning," Journal of Engineering for Gas Turbines and Power, Vol. 120, No. 1, 1998, pp. 115-119.

${ }^{7}$ Castanier, M. P., Ottarsson, G., and Pierre, C., "A Reduced-Order Modeling Technique for Mistuned Bladed Disks," Journal of Vibration and Acoustics, Vol. 119, No. 3, 1997, pp. 439-447.

${ }^{8}$ Yang, M. T. and Griffin, J. H., "A Reduced-Order Model of Mistuning Using a Subset of Nominal System Modes," Journal of Engineering for Gas Turbines and Power, Vol. 123, 2001, pp. 893-900.

${ }^{9}$ Petrov, E. P., Sanliturk, K. Y., and Ewins, D. J., "A New Method for Dynamic Analysis of Mistuned Bladed Disks Based on the Exact Relationship Between Tuned and Mistuned Systems," Journal of Engineering for Gas Turbines and Power, Vol. 124, No. 3, 2002, pp. 586-597.

${ }^{10}$ Lim, S., Bladh, R., Castanier, M. P., and Pierre, C., "A Compact, Generalized Component Mode Mistuning Representation for Modeling Bladed Disk Vibrations," Proceedings of the 44th AIAA/ASME/ASCE/AMS Structures, Structural Dynamics and Material Conference, Vol. 2, AIAA, Reston, VA, 2003, pp. 1359-1380.

${ }^{11}$ Castanier, M. P. and Pierre, C., "Consideration on the Benefits of Intentional Blade Mistuning for the Forced Response of Turbomachinery Rotors for the Forced Response of Turbomachinery Rotors," Analysis and Design Issues for Modern Aerospace Vehicles, Vol. AD-55, American Society of Mechanical Engineers, New York, 1997, pp. 419-425.

12 Judge, J. A., Pierre, C., and Mehmed, O., "Experimental Investigation of Mode Localization and Forced Response Amplitude Magnification for a Mistuned Bladed Disk," Journal of Engineering for Gas Turbines and Power, Vol. 123, 2001, pp. 940-950.

${ }^{13}$ Bladh, R., Pierre, C., Castanier, M. P., and Kruse, M. J., "Dynamics Response Predictions for a Mistuned Industrial Turbomachinery Rotor Using Reduced-Order Modeling," Journal of Engineering for Gas Turbines and Power, Vol. 124, No. 2, 2002, pp. 311-324.

${ }^{14}$ Judge, J. A., Experimental Investigation of the Effects of Mistuning on Bladed Disk Dynamics, Ph.D. thesis, The University of Michigan, Ann Arbor, MI, 2002.

${ }^{15}$ Pierre, C., Judge, J., Ceccio, S. L., and Castanier, M. P., "Experimental Investigation of the Effect of Random and Intentional Mistuning on the Vibration of Bladed Disks," Proceedings of the 7th National Turbine Engine High Cycle Fatigue Conference, Palm Beach Gardens, FL, 2002.

${ }^{16}$ Lim, S. H., Pierre, C., and Castanier, M. P., "Mistuning Identification and Reduced-Order Model Updating for Bladed Disk Based on a Component Mode Mistuning Technique," Proceedings of the 9th National Turbine Engine High Cycle Fatigue Conference, Pinehurst, North Carolina, 2004.

${ }^{17}$ Lim, S.-H., Dynamic Analysis and Design Strategies for Mistuned Bladed Disks, Ph.D. thesis, The University of Michigan, Ann Arbor, 2005.

${ }^{18} \mathrm{Li}$, J., Pierre, C., and Ceccio, S. L., "Validation of a New Technique for Mistuning Identification and Model Updating Based on Experimental Results for an Advanced Bladed Disk Prototype," NATO Paper RTO-MP-AVT-121-P-36, Proceedings of the NATO AVT-121 Symposium on Evaluation, Control and Prevention of High Cycle Fatigue in Gas Turbine Engines for Land, Sea and Air Vehicles, Granada, Spain, 2005.

${ }^{19}$ Gumbel, E. J., Statistics of Extremes, Columbia University Press, New York, NY, 1958.

${ }^{20}$ Gastillo, E., Extreme Value Theory in Engineering, Academic Press, Boston, MA, 1988.

${ }^{21}$ Judge, J. A., Pierre, C., and Ceccio, S. L., "Identification of Mistuning in Blisks," Proceedings of the 6th National Turbine Engine High Cycle Fatigue Conference, Jacksonville, FL, 2001.

22 Judge, J. A., Ceccio, S. L., and Pierre, C., "Traveling-Wave Excitation and Optical Measurement Techniques for NonContacting Investigation of Bladed Disks Dynamics," Shock and Vibration Digest, Vol. 35, No. 3, 2003, pp. $183-190$. 